\title{
Histology-Based Blood Leukocyte Profiling Reveals Parallel Th2 and Th17 Signatures in Seasonal Allergic Rhinitis
}

\section{Premkumar Siddhuraj}

Lund University

Franziska U Nordström

Lunds Universitet

Prajakta Jogdand

Lunds Universitet

Gayathri Elongovan

Lunds Universitet

Michiko Mori

Lunds Universitet

Leif Bjermer

Lunds Universitet

Lennart Greiff

Lunds Universitet

Jonas S Erjefält ( $\square$ jonas.erjefalt@med.lu.se )

Lunds Universitet https://orcid.org/0000-0002-6716-3010

\section{Research}

Keywords: allergy, biomarker, peripheral leukocytes, allergic rhinitis, Th17, basophils

Posted Date: February 18th, 2020

DOI: https://doi.org/10.21203/rs.2.23865/v1

License: (1) (1) This work is licensed under a Creative Commons Attribution 4.0 International License. Read Full License 


\title{
Histology-Based Blood Leukocyte Profiling Reveals Parallel Th2 and Th17 Signatures in Seasonal Allergic Rhinitis
}

\author{
Premkumar Siddhuraj ${ }^{1}$, Franziska U Nordström ${ }^{2,3}$, Prajakta Jogdand ${ }^{1}$, Gayathri \\ Elangovan $^{1}$, Michiko Mori ${ }^{1}$, Leif Bjermer ${ }^{4}$, Lennart Greiff ${ }^{2,3}$, and Jonas S Erjefält ${ }^{1,4}$ \\ ${ }^{1}$ Department of Experimental Medical Science, Lund University, Lund, Sweden \\ ${ }^{2}$ Department of Otorhinolaryngology, Head and Neck Surgery, Skåne University Hospital, \\ Lund, Sweden \\ ${ }^{3}$ Department of Clinical Sciences, Lund University, Lund, Sweden \\ ${ }^{4}$ Department of Respiratory Medicine and Allergology, Skåne University Hospital, Sweden
}

Corresponding author and requests for reprints should be addressed to:

Jonas Erjefält, Prof., e-mail: jonas.erjefalt@med.lu.se

Airway Inflammation Unit, Dept. of Experimental Medical Science,

BMC D12, Lund University, SE-22184, Lund, Sweden

Phone: +46 46222 0960, Fax: +46 462113417

Sources of support: The Heart \& Lung Foundation, Sweden, the Swedish Medical Research Council, the Swedish Asthma and Allergy Associations Research Foundation, and the Swedish Cancer and Allergy Foundation.

Running head: Histology-based blood leukocyte profiling in allergic rhinitis 


\begin{abstract}
Background: Allergic rhinitis (AR) is one of the most common medical conditions in the westernized world. Recent data suggest AR to be far more immunologically complex than the archetypical allergic Th2-driven eosinophilic inflammation and new methodological approaches are needed to decode this complexity.

Methods: This study explores a novel histology-based analysis of circulating leukocytes for detailed profiling of immune cells using routine clinical blood samples. In brief, leukocytes were purified with minimal ex-vivo artefacts, embedded into agarose-paraffin pellets and sectioned for cutting-edge immunohistochemistry-based immune cell profiling. Blood leukocyte mapping was performed in 16 patients with seasonal AR outside and during the birch pollen season.
\end{abstract}

Results: Our methodological feasibility test confirmed that the $>5000$ cross sectioned leukocytes typically present in a pellet section had well preserved morphology and cell marker epitopes, allowing for robust quantitative analysis of immune-stained slides. Blood leukocyte samples collected during the allergen season had statistically higher levels of markers for eosinophils, neutrophils, monocytes and CD8 lymphocytes compared to the off-season baseline. No change was observed for CD20 B-lymphocytes, total CD3 T cells and basophils. Subclassification of CD4+ T-helper cells demonstrated a parallel and significant expansion of Th2 and Th17 cells during the pollen season, while Th1 cells remained unchanged. Whereas absolute basophils numbers were unaltered, a significant increase of the basophil markers GATA2 and CPA3 was observed during the pollen season.

Conclusions: Apart from representing a positive method feasibility validation, our study provides further evidence of complex and parallel Th2 and Th17 immune signatures in seasonal AR. Our data also forward GATA2 and basophil CPA3 as potential biomarkers for ongoing allergic inflammation. It is thus proposed that the present histology-based approach, with its broad applicability, represents a powerful tool for decoding systemic immune alterations and guide novel biomarker strategies for improved personalized medication.

Key words: allergy, biomarker, peripheral leukocytes, allergic rhinitis, Th17, basophils 


\section{BACKGROUND}

Allergic rhinitis (AR) represents one of the most common chronic medical conditions for seeking medical care(1). The disease is largely considered an IgE-mediated disorder with an allergic and Th2-mediated eosinophil-rich inflammation in the nasal mucosa as the main immunopathological feature(1-3). The typical symptoms are nasal itching/sneezing, rhinorrhoea, and nasal congestion. Globally, more than 400 million patients suffer AR and still today the disease is to a large extent underdiagnosed and undertreated. The pharmacological treatment for allergic rhinitis includes intranasal corticosteroids, oral and topical antihistamines and intranasal cromolyn(4-6). Additional treatment options are immunotherapy and, more recently, biologics targeting $\operatorname{IgE}$ (omalizumab)(7) or type 2 cytokines and their receptors (e.g mepolizumab, reslizumab, and benralizumab). Naturally, the use of costly biologics is predominantly for AR patients with concomitant type 2 and eosinophilic asthma and/or nasal polyposis. However, with more long-term safety data and the expected future price reduction, biologics will likely become a treatment option for a larger group of patients with AR. Indeed, improved treatment regiments are needed, because even with the best current pharmacotherapy up to $20 \%$ of the patients remain highly symptomatic.

One important factor to optimize treatment is the introduction of improved biomarkers. In particular, in the emerging era of personalized medicine, novel biomarkers are needed to inform about the immunological and inflammatory nature underlying the symptoms in individual patients. This approach is highly warranted since recent data has forwarded AR as a more immunologically complex disease than previously thought $(4,8)$. Thus, although the archetype allergic Th2 and IgE-driven eosinophilic inflammation plays an important role(2, 9), other separate and/or parallel immune responses may also contribute to the immunopathology. For example, it has recently been observed that neutrophil responses may be a significant feature of AR (10). Other reports provide evidence that also Th17 immune responses may contribute(11).

A range of biomarkers have been tested for use in aiding diagnosis, monitor disease activity and inform about inflammatory status in subjects with allergic rhinitis (8, 12-14). Plasma exudation, which is a hallmark of inflammation, can be analysed as extravasated plasma proteins like alpha-2 macroglobulin in nasal lavage fluids to yield a good umbrella parameter of ongoing inflammation $(15,16)$. Nasal lavage, or collection of more concentrated nasal surface secretions, have also been used to analyse individual immune cells or cytokines (17). However, 
although informative, the use of such sampling is limited in clinical practice by its relative invasive nature and by technical challenges involved in normalising against variations in fluid recovery and by variations in nasal mucosal surface liquid viscosity. Assessment of routine blood samples overcome these challenges and has, despite only being a systemic measure, been used to monitor immunological markers and circulating leukocytes in AR (18-20). The assessment of peripheral blood leukocytes in AR has mainly been performed by flow cytometry (FACS). While this undoubtedly is a powerful and valuable method, tentative limitations include artefactual cell changes during the cell purification and that the cell marker to be analysed is limited and must be determined beforehand.

The present study explores a novel type of histology-based analysis of blood leukocytes as an alternative or complementary methodological approach for profiling circulating immune cells present in routine blood samples. Specifically, we test this approach on enriched blood leukocytes that have been subjected to minimal ex vivo handling before being fixed and embedded in paraffin for cutting-edge histological analysis. Comparisons are made between multiple leukocyte population markers in samples collected during and after the natural pollen season in patients with seasonal AR.

\section{METHODS}

\section{Patients and Clinical Sampling}

A total of 16 patients with seasonal AR were recruited to the study (mean age 25.6 years, range 20-39 years). Among the inclusion criteria was a history of seasonal AR for at least 2 years. Another requirement was a positive skin prick test to birch pollen allergen or other season-relevant allergen (15 out of the 16 patients were allergic to birch pollen and the total average wheal size was $5.9 \mathrm{~mm}$, range 2-50). Exclusion criteria were chronic nasal disease and a history of asthma. Although most patients had more or less strict seasonal rhinitis, individuals with a positive skin prick test to house dust mite or animal dander were allowed to participate if they did not present any perennial symptoms and if they were not exposed to allergenrelevant animals. The study was approved by the local ethics committee (Swedish ethical review board, Lund: Dnr 2016/166) and informed consent was obtained from all patients.

Leukocyte profiles were explored in peripheral blood samples that were collected during the Swedish birch pollen season in the spring. Comparisons were made to control off-season 
blood samples were obtained late in the autumn, several weeks after the last episode of the Swedish pollen season.

\section{Assessment of Symptoms}

Symptoms were recorded during the pollen season study period using validated diary $\operatorname{cards}(21,22)$. The recorded symptoms, i.e. rhinorrhoea, nasal blockage and sneezing, were scored on a four-graded scale: grade 0 , none; grade 1, mild; grade 2, moderate; and grade 3 , severe symptoms. For each day, a total nasal symptom score (TNSS) was calculated.

\section{Preparation of Blood Leukocyte Pellets}

To make blood leukocyte pellets we slightly modified a protocol that previously has been shown to result in minimal ex-vivo handling artifacts or handling-evoked accidental cell activation(23)(Figure 1A). In brief, within minutes after the collection the $8 \mathrm{ml}$ blood samples were immediately transferred into heparin tubes containing $16 \mathrm{ml}$ of $4 \%$ buffered formalin (Histolab-REF:02176, 4\%, pH7.4) and left for 1-hour at room temperature. To sediment red blood cells (RBC) from formalin-fixed whole blood, the blood sample was divided into two equal volumes of $12 \mathrm{ml}$ and each portion was transferred into a tube containing $5 \mathrm{ml}$ of dex$\operatorname{tran}(5 \%$ dextran $+3 \mathrm{~g}$ glucose in PBS $)$ and left for 1-hour at room temperature.

After 1-hour dextran-based RBC sedimentation, the leukocyte enriched plasma was carefully retrieved by a pipette and centrifuged at $1400 \mathrm{rpm}$ (at room temperature $27^{\circ} \mathrm{C}$ ) for 10 minutes. The obtained leukocyte pellet was resuspended into $4 \%$ formalin and transferred into a tube containing pre-warmed melted agarose (low melting agarose, Sigma-Aldrich A4018-10G) and again centrifuged at $1400 \mathrm{rpm}$ for 10 minutes (at $27^{\circ} \mathrm{C}$ ). The hardened agarose containing the leukocyte pellet was left in 4\% formalin for approximately 12 hours (overnight) at room temperature before being subjected to standard dehydration (SAKURA-Tissue-Tek VIP® 6 AI automated dehydration machine) and embedding into paraffin pellets.

\section{Immunohistochemistry}

Paraffin-embedded blood pellets were sectioned at $4 \mu \mathrm{m}$ thickness, and at least five blood pellet sections were placed per slide to create blood pellet micro array slides. As part of the antigen retrieval process, slides were baked at $60^{\circ} \mathrm{C}$ for 45 minutes and pretreated with $\mathrm{pH}$ low (pH 6) in an antigen retrieval solution (Dako-DM829) in Dako PT Link machine $\left(97^{\circ} \mathrm{C}\right.$ for 20 minutes; PT-200, Dako/Agilent, Glostrup, Denmark). 
A modified double and triple staining immunohistochemistry protocol was applied for simultaneous visualization of multiple immune cell markers(24, 25). Briefly, after antigen retrieval, endogenous peroxidase was blocked with peroxidase blocking reagent (Dako-DM821) for 10 minutes. Next, the slides were incubated with the primary antibody for 1 hour, washed extensively and thereafter incubated with an isotype-relevant secondary antibody coupled to a HRP-labeled polymer (Envision Flex-DM822 amplification kit) for 30 minutes before being subjected to a 10 minute incubation with HRP chromogen substrate. Excess remaining chromogen substrate and/or non-encapsulated HRP in the sections were denatured using 1:3 diluted denaturing solution (Biocare Medical- DNS 001L) for 5 minutes. After the denaturation step, a similar protocol round was repeated to achieve the double and triple staining. For double and triple staining, the following HRP chromogens were used; 3,3'-Diaminobenzidine (DAB-Dako-DM827, brown precipitate), vina green (Biocare-BRR807BC) and deep space black (Biocare- BRI4015 H, L). Finally, the slides were stained by hematoxylin (Htx) nucleus counterstaining and mounted in pertex (Pertex-Histolab). Information about the primary cell marker antibodies are presented in Table 1. Control experiments where the primary antibody was replaced with a non-relevant isotype control did not result in any staining. Importantly, for the comparison between off-season and season marker values, all sample preparations were identical and off-seasonal and seasonal samples were IHC stained and analyzed simultaneously.

\section{Digitalization of Immunostained Leukocyte Pellets and Computerized Image Analysis}

An automated Olympus VS-120 Virtual Slide Microscope was used for high resolution digitalization (20X lens magnification) of the entire section area from all multi-stained blood pellet sections. In each cell pellet the immunoreactivity for each stained cell marker (i.e. the detection chromogens), as well as the background Htx-stained cell nuclei, were automatically detected by color and spectral-based segmentation by computerized image analysis (Visiomorph software, Visopharm, Denmark) (Figure 1 D). Finally, for each cell marker, the total area (pixels) of the corresponding chromogen was calculated.

The areas of nuclei and immunostainings were combined to obtain the total area of blood pellet. Finally, the area of each particular marker was divided with the total cell pellet area and converted into a percentage of immunoreactivity.

In order to automatically detect double stained Th1, Th2, and Th17 CD4+ lymphocytes, each nucleus with positive staining for the relevant nuclear transcription factor (T-bet, GATA3, 
and ROR- $\gamma \mathrm{t}$, respectively) were detected by color segmentation. Next, any transcription factor-positive nucleus that was in direct spatial contact with surrounding $\mathrm{CD} 4^{+}$immunoreactivity was automatically reclassified as the corresponding Th subset (Figure 1 E).

\section{Statistics}

All the data were presented as scattergrams and mean values. As all the data were from the same sample population, The Wilcoxon test for paired observations was used for statistical analysis in Prism 8. The Pearson correlation coefficient analysis was performed to investigate correlations between cell markers, or between markers and symptom scores.

\section{RESULTS}

\section{Method Optimization and Validation}

Initial tests revealed that the current protocol of generating paraffin blood leukocyte pellets for histological analysis resulted in a well-preserved morphology of the cells as inspected after routine hematoxylin staining. Heat-induced antigen retrieval prior to IHC had a slightly negative effect on the morphology. Thus, initial studies, with different combinations of programmed time and temperature curves were tested in order to yield the combination that was best suited morphology preservation, robust IHC staining and computerized image analysis (Figure 1B-C). Our preliminary explorations also revealed that each cross sectioned pellet typically contained cross section planes of 5000 - 7000 individual leukocytes.

Using the optimized protocol with $97{ }^{\circ} \mathrm{C}$ HIER for 20 minutes, freshly cut sections from all season and off-season blood samples were simultaneously subjected to immunohistochemical staining and computerized image analysis. Importantly, despite that our type of quantification is conceptually different from conventional routine differential cell counts, the rough overall proportions of cell markers representing the major cell types was fairly similar to the expected overall leukocyte composition (Figure $2 B$ ). Further, of relevance to allergic diseases, routine clinical eosinophil differential counts correlated with the present histology-based enumeration of immunoreactivity for the eosinophil cationic protein marker EG2 (Figure 2A).

\section{Altered Blood Cell Marker Immunoreactivity During the Allergen Season}

Next, we performed a paired analysis comparing off-season (autumn) and allergic season (spring) immunoreactivity values for selected basic cell populations. Our data revealed statistically elevated levels of EG2 (eosinophils), CD68 (monocytes), and MPO (neutrophils) dur- 
ing the spring compared to paired off-season autumn values (Figure 2 C-I). No statistical change could be observed for the basophil marker proMBP1, the pan $\mathrm{T}$ cell marker CD3, or the B-lymphocyte marker CD20 (Figure 2).

\section{Increased Th2 and Th17 Blood Signatures}

Assessment of T-lymphocyte subpopulation, including transcription factor-defined CD4+ Thelper subsets, demonstrated a statistically elevated value for CD8 immunoreactivity (i.e. cytotoxic T cells) compared to off-season values. Among the Th cell types $\mathrm{CD}^{+}{ }^{+}, \mathrm{GATA}^{+}$double positive Th2 cells and $\mathrm{CD}^{+}, \mathrm{ROR} \mathrm{t}^{+}$Th17 cells increased during the pollen season, whereas no such change was observed for $\mathrm{CD}^{+}$, Tbet $^{+}$Th1 cells (Figure 3).

\section{Basophil-Related Markers}

Basophils are classically linked to allergic disorders. Interestingly, although no statistical seasonal increase was observed for the basophil markers pMBP1 (Figure 2F), a robust statistical increase was observed for the basophil transcription factor GATA2 (Figure 4 C). A subset of the basophils has been described to also contain the granule proteases CPA3 and tryptase (26, 27). During the allergen season elevated blood leukocyte CPA3 but not tryptase could be detected together with a non-statistical trend towards increased surface bound IgE (Figure 4A,D$E)$.

\section{Cell Marker Correlations}

During the allergen season many cell markers seemed to be expressed independently within individual patients. However, a statistical correlation was present between EG2/eosinophils and the basophil marker GATA2 (Figure 5A). Intriguingly, a surprisingly strong correlation was also found between Th2 and Th17 lymphocytes (Figure 5B). Although our study only included a limited number of patients it allowed for some insight into the correlation between autumn and spring values among the various IHC markers. Some markers, such as blood leukocyte tryptase (i.e. basophil tryptase), EG2/eosinophils and the B cell marker CD20, was of similar levels within patients examined before and after the pollen season.

Despite that many of the cell markers were statistically increased during the spring pollen season, there was no clear correlations between leukocyte markers and the spring symptom scores. 


\section{DISCUSSION}

The heterogeneous nature of the inflammation AR, and the emergence of new selective treatment options, call for improved ways to explore the immunological heterogeneity without invasive nasal sampling. This study presents such an approach and demonstrates that histological analysis of blood leukocyte pellets can be used for mapping circulating immune cell profiles. The fact that many immune cell parameters were statistically increased during the allergen season compared to off-season show the capacity of the present approach to monitor clinically relevant changes in inflammation status. Of particular interest was our finding of increased parallel Th2, Th17, neutrophil, and eosinophil responses during the allergen season. Our data also reveal a potential use of the basophil-associated markers GATA2 and CPA3 as markers of ongoing allergic inflammation. On a general note, the present approach for histology-based leukocyte mapping is certainly not restricted to allergic rhinitis and has bearings on most other inflammatory conditions.

From a methodological perspective, it is important to stress that the present approach to monitor blood immune cell marker is conceptually different from standard assessments of the leukocyte composition in peripheral blood. Thus, whereas FACS analysis and blood differential cell counts in routine clinical labs measure the proportions of whole cells, our approach rather measures the normalized area of actual staining (i.e. immunoreactivity). Naturally, the stained marker area per marker-positive cell area may differ between different types of markers. For example, the presently used nuclear transcription factors, cell membrane or cytoplasmic granule markers differ in respect to how much area of the entire cell profile that is actually stained. From this, together with marker differences in antibody dilution and staining intensity, it should be expected that the relative proportions between the present cell identification markers do not correspond to the exact proportions of cell numbers. Despite this, a surprisingly good correlation was achieved between the overall leukocyte proportions and between eosinophil numbers determined by a routine clinical lab and our parameter of EG2 immunoreactivity.

The present study demonstrates clearly that our methodological approach has the sensitivity to detect multiple allergen-evoked changes among circulating blood leukocytes. Among these, the significant eosinophil and Th2 responses were expected. Indeed, eosinophilia, which is a 
hallmark of $\mathrm{AR}$, and is thought to be driven by an underlying Th2 response. Of immunopathological relevance, allergen-induced immune responses not only stimulate eosinophil infiltration into the nasal mucosa but also their activation and cytotoxic mediator release through piecemeal degranulation (PMD) and programmed cytolysis (3, 21, 28). Perhaps seemingly more surprising was our observation of very robust increases in Th17 cells and MPO immunoreactivity, suggesting a significant IL-17 driven neutrophil response. This suggestion is further supported by the fact that IL17 cytokines like IL17A and IL17F stimulate immune cells and epithelial cells to release the neutrophil chemokines G-SCF and IL8/CXCL8 $(29,30)$. Taken together, our findings thus agree with the several studies that suggest neutrophilia and mixed eosinophilic and neutrophilic phenotypes as a significant feature in $\operatorname{AR}(10,19,31)$. It is likely that the mechanism behind co-emergence of eosinophilia and neutrophilia in AR are multifactorial. Interestingly, among many alternatives, a recent report suggests that neutrophils in rhinitis patients may not only be down-stream effector cells but may also contribute to the allergic eosinophilia by priming T-cells to recruit eosinophils (10).

Basophils have been implicated in allergic diseases $(26,32)$ and basophil-associated biomarkers have an important role to e.g. predict response to immunotherapy (33-35). Basophil activation status in the blood is commonly analyzed by FACS as a translocation of CD63, CD69 and CD203c to the cell surface(36-38). Unfortunately, the present type of histological analysis cannot reliably differentiate between cytosol and membrane immunoreactivity. Hence, we explored five alternative basophil-associated markers; pMPB1, GATA2, CPA3, tryptase and surface bound IgE. While several of these may be expressed on other cells in inflamed tissues, they will be more of less unique to basophils in circulating blood(26). For example, most mast cells express GATA2, tryptase and surface bound IgE, and a significant proportion also expresses CPA3. However, mature mast cells are not present in the blood and mast cell progenitors are so exceedingly rare that they cannot have contributed to our blood analysis. The marker prp-MPB1 has been forwarded as a basophil pan marker but may also be present in eosinophil progenitors. Eosinophil progenitors, which are also expected to increase during the allergen season, also express GATA2 and may thus be regarded as a confounding cell in our assessment of basophils. However, when staining for $\mathrm{CD} 34^{+}$progenitor cells these were present in exceedingly low numbers (data not shown). Hence, neither eosinophils nor mast cell progenitors could have contributed significantly to our basophil marker analysis. Among these markers CPA3 and GATA2 values were significantly higher in-season than during off-season conditions. Although the biological background to this remain unknown, these 
markers may therefore be added to the list of potential blood biomarkers for assessing allergic inflammation.

It should be noted that the main objective of the present study was to explore the potential and feasibility of the present histology-based approach for advanced blood leukocyte profiling of routine blood samples. We are aware that the number of the patients is limited and relatively small for this type of biomarker validation. We also noted that there was a rather marked variation among the included patients with regard to their symptom profiles. Nevertheless, the fact that our study design allows for paired analysis, and the fact that we did observe clear statistical season - off-season differences for several parameters, underscore the potential of this method. Indeed, one advantage over most other techniques is that the paraffin-embedded leukocyte pellets can be stored at room temperature for years and recut to make any type of follow-up analyses with a new round of immune markers. Although not explored here, advanced computerized image analysis may also be used to generate "FACS-like" data measuring staining intensity for all of the thousands individual cells typically present within a leukocyte pellet section. Naturally, the present methodology is fairly technically demanding and may not readily be suited for routine immune screening in a clinical setting. In spite of this, there is certainly an important role for more in depth immune cell profiling in the general goal to decode the immunological heterogeneity among AR patients. The type of data that is generated may also provide an important guidance to develop less complicated assays that can be tailored for implementation in real life clinical settings.

\section{Conclusions}

In summary, we present here a novel methodological approach allowing a robust and multifaceted mapping of circulating leukocyte population in routine blood samples. Apart from representing a positive feasibility validation of this approach, our study provides further evidence of complex and parallel Th2 and Th17 immune signatures in seasonal AR and a surprisingly robust upregulation of the blood basophil markers GATA2 and CPA3. Further studies applying these and other markers to larger patient cohorts is suggested to provide valuable insight into the immunological complexity of allergic rhinitis and other inflammatory conditions. 


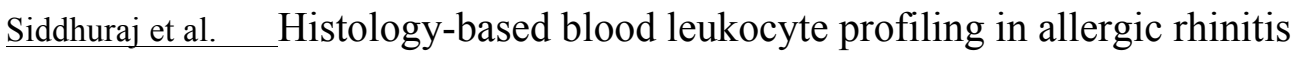

Table 1. Immune Cell Markers and Primary Antibodies

\begin{tabular}{|c|c|c|c|c|}
\hline Antigen & Cell Type & Species & Dilution & Manufacturer and Clone \\
\hline CD4 & Pan T- helper lymphocytes & Mouse & $1: 50$ & Novacastra-NCL-L-CD4-368 \\
\hline T-Bet & Th1 cells (if CD4-positive) & Rabbit & $1: 1500$ & Santa Cruz -H210 \\
\hline GATA3 & Th2 cells (if CD4-positive) & Mouse & $1: 1500$ & BD Pharmagen-L50-823 \\
\hline $\mathrm{ROR}_{y} \mathrm{t}$ & Th17 cells (if CD4-positive) & Rabbit & $1: 50$ & Abcam-ab207082 \\
\hline MPO & Neutrophils & Rabbit & $1: 30000$ & Dako-A0398 \\
\hline CD20 & B-lymphocytes & Mouse & $1: 1200$ & Dako-M0755 \\
\hline CD3 & Pan $\mathrm{T}$ lymphocytes & Rabbit & $1: 120$ & Biocare \\
\hline EG2 & Eosinophils & Mouse & $1: 1000$ & Pharmacia. / EG2 \\
\hline GATA2 & Basophils (tissue mast cells) & Rabbit & $1: 1200$ & Novus- NMP1-82581 \\
\hline CD68 & Macrophages / monocytes & Mouse & $1: 50$ & Novacastra-NCL-L-CD163 \\
\hline IgE & Cells with surface bound $\operatorname{IgE}$ & Mouse & $1: 1000$ & DAKO \\
\hline pMBP1 & Basophils & Mouse & $1: 50$ & Biolegend \\
\hline CD8 & Cytotoxic T lymphocytes & Mouse & $1: 50$ & DAKO-C0/144B \\
\hline CPA3 & Basophils (+tissue MCs) & Rabbit & $1: 1000$ & Atlas-HPA008689 \\
\hline Tryptase & Basophils (+tissue MCs) & Mouse & $1: 1000$ & Millipore-MAB1222A \\
\hline
\end{tabular}




\section{Legends}

Figure 1. Schematic overview of the blood leukocyte pellet preparation (A). After blood sampling and a brief pre-fixation, cells are separated by dextran sedimentation (1). Layers containing leukocytes are collected and the white blood cells are spun down into pre-warmed melted agarose (2), which after being allowed to solidify and subjected to routine $4 \%$ formaldehyde fixation is embedded into paraffin (3) and cut into sections used for detection of multiple leukocyte markers by immunohistochemistry (exemplified in 4). Selection of optimal low $\mathrm{pH}$ heat-induced antigen retrieval conditions (B) and representative example of Htxstained leukocytes amidst the few remaining erythrocytes (C). Example of detection and segmentation strategies for computerized quantitative image analysis (D-E). In panel $\mathrm{E}$ the brown ROR $\gamma \mathrm{t}$ - positive nuclei up to the left is touching CD4 immunoreactivity and is thus reclassified as a Th17 cell.

Figure 2. Correlation between clinical routine eosinophil cell count and computerized quantification of the eosinophil granule marker EG2 in blood leukocyte pellets (A). The overall composition and relative proportion of immunoreactivity for common leukocyte identity markers (B). Quantitative data of pairwise measurements of immunoreactive area in blood leukocyte pellets collected off-season/autumn and during the spring pollen season (C-I). Data shown are for major leukocyte population markers. * and *** denote a significance level of $\mathrm{p}$ $<0.05$ and $\mathrm{p}<0.001$, respectively. Correlation in $\mathrm{A}$ is analyzed by Pearson correlation coefficient analysis.

Figure 3. Quantitative T-helper subset data of pairwise autumn and spring measurements for each individual patient (A,C,F). Th1, Th2, and Th17 populations were identified by double staining for CD4 and their corresponding transcription factor; i.e. T-bet, GATA3 and ROR $\gamma \mathrm{t}$ $(B, D, E, G) . *$ and $* *$ denote a significance level of $\mathrm{p}<0.05$ and $\mathrm{p}<0.01$, respectively.

Figure 4. Pairwise leukocyte pellet data for the basophil-associated markers IgE, GATA2, CPA3 and Tryptase (A,C-E). ** and *** denote a significance level of $\mathrm{p}<0.01$ and $\mathrm{p}<0.001$, respectively. Representative example images of IgE-stained blood leukocytes (B). Triple immunofluorescence staining exemplifying a basophil, detected with the basophil marker BB1, and which is double positive for the granule proteases CPA3 and tryptase. 
Figure 5. Scattergrams demonstrating the correlation between blood leukocyte pellet levels of eosinophils (EG2 immunoreactivity) and the basophil-associated transcription factor GATA2 (x axis in A). Correlations between blood pellet Th2 and Th17 cells during the pollen season are presented in panel B. The level of statistical correlation was tested with Pearson correlation coefficient analysis.

\section{DECLARATIONS}

\section{Abbreviations}

AR: Allergic rhinitis; TNSS: total nasal symptom score; HIER: heat-induced epitope retrieval; ISH: in situ hybridization; HRP: horse radish peroxidase; IHC: immunohistochemistry; PBS; phosphate buffered saline; CPA3: carboxypeptidase 3; CD: cluster of differentiation; Th: T helper; pMBP: pro major basic protein; MPO: myeloperoxidase; MC: mast cells; IgE: Immunoglobulin $\mathrm{E}$

\section{Acknowledgements}

We thank Karin Janser and Britt-Marie Nilsson and Daisy Bornesund for coordinating the sample preparations and skillful technical assistance. We would also like to thank Charlotte Cervin-Hoberg and Lena Glantz Larsson for organizing the patient recruitment and blood sampling.

\section{Author's Contributions}

LG, FN, and JE, conceived and designed the clinical study design: LG and FN coordinated the patient selection and clinical sampling. PS, MM and JE designed the histological studies. PJ, MM, PS, and JE validated the leukocyte preparation protocol. PS, GE, and MM performed the histological experiments and quantifications. JE and PS analysed and interpreted the data and wrote the manuscript. LG, FN, and LB contributed with data interpretation and manuscript editing. All authors read and approved the final manuscript.

\section{Ethics Approval and Consent to Participate}

The present study was approved by the local ethics committee (Swedish ethical review board, Lund: Dnr 2016/166) and informed consent was obtained from all patients. 
Siddhuraj et al. Histology-based blood leukocyte profiling in allergic rhinitis

\section{Consent for Publication}

All authors approved to the publication of the present study

\section{Funding}

The present study was funded by project grants from the Swedish Medical Research Council, the EU Interreg program, the Swedish Heart \& Lung foundation, the Asthma and Allergy foundation as well as the Asthma and Cancer foundation.

\section{Availability of Data and Materials}

The datasets used and/or analyzed during the current study are available from the corresponding author on reasonable request.

\section{Competing Interests}

The authors declare that they have no competing interests. 
Siddhuraj et al. Histology-based blood leukocyte profiling in allergic rhinitis

\section{References}

1. Greiner AN, Hellings PW, Rotiroti G, Scadding GK. Allergic rhinitis. Lancet 2011; 378: 2112-2122.

2. Borish L. Allergic rhinitis: systemic inflammation and implications for management. $J$ Allergy Clin Immunol 2003; 112: 1021-1031.

3. Erjefalt JS, Greiff L, Andersson M, Matsson E, Petersen H, Linden M, Ansari T, Jeffery PK, Persson CG. Allergen-induced eosinophil cytolysis is a primary mechanism for granule protein release in human upper airways. Am J Respir Crit Care Med 1999; 160: 304-312.

4. Meng Y, Wang C, Zhang L. Recent developments and highlights in allergic rhinitis. Allergy 2019; 74: 2320-2328.

5. Heffler E, Brussino L, Del Giacco S, Paoletti G, Minciullo PL, Varricchi G, Scadding G, Malvezzi L, De Virgilio A, Spriano G, Puggioni F, Fornero M, Rolla G, Canonica GW. New drugs in early-stage clinical trials for allergic rhinitis. Expert Opin Investig Drugs 2019; 28: 267-273.

6. Klimek L, Mullol J, Hellings P, Gevaert P, Mosges R, Fokkens W. Recent pharmacological developments in the treatment of perennial and persistent allergic rhinitis. Expert Opin Pharmacother 2016; 17: 657-669.

7. Vashisht P, Casale T. Omalizumab for treatment of allergic rhinitis. Expert Opin Biol Ther 2013; 13: 933-945.

8. Segboer CL, Fokkens WJ, Terreehorst I, van Drunen CM. Endotyping of non-allergic, allergic and mixed rhinitis patients using a broad panel of biomarkers in nasal secretions. PLoS One 2018; 13: e0200366.

9. Gelfand EW. Inflammatory mediators in allergic rhinitis. J Allergy Clin Immunol 2004; 114: S135-138. 
Siddhuraj et al. Histology-based blood leukocyte profiling in allergic rhinitis

10. Arebro J, Ekstedt S, Hjalmarsson E, Winqvist O, Kumlien Georen S, Cardell LO. A possible role for neutrophils in allergic rhinitis revealed after cellular subclassification. Sci Rep 2017; 7: 43568.

11. Gu ZW, Wang YX, Cao ZW. Neutralization of interleukin-17 suppresses allergic rhinitis symptoms by downregulating Th2 and Th17 responses and upregulating the Treg response. Oncotarget 2017; 8: 22361-22369.

12. Wang WY, Boot JD, Mascelli MA, Gerth van Wijk R, Diamant Z. Comparison of biomarkers between allergic rhinitis only and allergic rhinitis with concomitant asthma. Allergy 2009; 64: 1102-1103.

13. Cipriani F, Tripodi S, Panetta V, Perna S, Potapova E, Dondi A, Bernardini R, Caffarelli C, Casani A, Cervone R, Chini L, Comberiati P, De Castro G, Miraglia Del Giudice M, Dello Iacono I, Di Rienzo Businco A, Gallucci M, Giannetti A, Mastrorilli C, Moschese V, Pelosi S, Sfika I, Varin E, Villella V, Zicari AM, Brindisi G, Ricci G, Matricardi PM, Italian Pediatric Allergy N. Early molecular biomarkers predicting the evolution of allergic rhinitis and its comorbidities: A longitudinal multicenter study of a patient cohort. Pediatr Allergy Immunol 2019; 30: 325-334.

14. Han MW, Kim SH, Oh I, Kim YH, Lee J. Serum IL-1beta can be a biomarker in children with severe persistent allergic rhinitis. Allergy Asthma Clin Immunol 2019; 15: 58.

15. Greiff L, Andersson M, Erjefalt JS, Persson CG, Wollmer P. Airway microvascular extravasation and luminal entry of plasma. Clin Physiol Funct Imaging 2003; 23: 301-306.

16. Persson CG, Erjefalt JS, Greiff L, Andersson M, Erjefalt I, Godfrey RW, Korsgren M, Linden M, Sundler F, Svensson C. Plasma-derived proteins in airway defence, disease and repair of epithelial injury. Eur Respir $J$ 1998; 11: 958-970.

17. Greiff L, Ahlstrom-Emanuelsson C, Alenas M, Almqvist G, Andersson M, Cervin A, Dolata J, Lindgren S, Martensson A, Young B, Widegren H. Biological effects and clinical 
Siddhuraj et al. Histology-based blood leukocyte profiling in allergic rhinitis

efficacy of a topical Toll-like receptor 7 agonist in seasonal allergic rhinitis: a parallel group controlled phase IIa study. Inflamm Res 2015; 64: 903-915.

18. Diamant Z, Boot JD, Mantzouranis E, Flohr R, Sterk PJ, Gerth van Wijk R. Biomarkers in asthma and allergic rhinitis. Pulm Pharmacol Ther 2010; 23: 468-481.

19. Jordakieva G, Jensen-Jarolim E. The impact of allergen exposure and specific immunotherapy on circulating blood cells in allergic rhinitis. World Allergy Organ J 2018; 11: 19 .

20. Malm-Erjefalt M, Persson CG, Erjefalt JS. Degranulation status of airway tissue eosinophils in mouse models of allergic airway inflammation. Am J Respir Cell Mol Biol 2001; 24: 352-359.

21. Ahlstrom-Emanuelsson CA, Greiff L, Andersson M, Persson CG, Erjefalt JS. Eosinophil degranulation status in allergic rhinitis: observations before and during seasonal allergen exposure. Eur Respir J 2004; 24: 750-757.

22. Greiff L, Ahlstrom-Emanuelsson C, Bahl A, Bengtsson T, Dahlstrom K, Erjefalt J, Widegren H, Andersson M. Effects of a dual CCR3 and H1-antagonist on symptoms and eosinophilic inflammation in allergic rhinitis. Respir Res 2010; 11: 17.

23. Malm-Erjefalt M, Greiff L, Ankerst J, Andersson M, Wallengren J, Cardell LO, Rak S, Persson CG, Erjefalt JS. Circulating eosinophils in asthma, allergic rhinitis, and atopic dermatitis lack morphological signs of degranulation. Clin Exp Allergy 2005; 35: 1334-1340. 24. Bergqvist A, Andersson CK, Mori M, Walls AF, Bjermer L, Erjefalt JS. Alveolar Thelper type-2 immunity in atopic asthma is associated with poor clinical control. Clin Sci (Lond) 2015; 128: 47-56.

25. Pretolani M, Bergqvist A, Thabut G, Dombret MC, Knapp D, Hamidi F, Alavoine L, Taille C, Chanez P, Erjefalt JS, Aubier M. Effectiveness of bronchial thermoplasty in patients 
Siddhuraj et al. Histology-based blood leukocyte profiling in allergic rhinitis

with severe refractory asthma: Clinical and histopathologic correlations. J Allergy Clin Immunol 2017; 139: 1176-1185.

26. Korosec P, Turner PJ, Silar M, Kopac P, Kosnik M, Gibbs BF, Shamji MH, Custovic A, Rijavec M. Basophils, high-affinity IgE receptors, and CCL2 in human anaphylaxis. $J$ Allergy Clin Immunol 2017; 140: 750-758 e715.

27. Samorapoompichit P, Kiener HP, Schernthaner GH, Jordan JH, Agis H, Wimazal F, Baghestanian M, Rezaie-Majd A, Sperr WR, Lechner K, Valent P. Detection of tryptase in cytoplasmic granules of basophils in patients with chronic myeloid leukemia and other myeloid neoplasms. Blood 2001; 98: 2580-2583.

28. Erjefalt JS, Persson CG. New aspects of degranulation and fates of airway mucosal eosinophils. Am J Respir Crit Care Med 2000; 161: 2074-2085.

29. Fossiez F, Banchereau J, Murray R, Van Kooten C, Garrone P, Lebecque S. Interleukin17. Int Rev Immunol 1998; 16: 541-551.

30. Persson CG, Erjefalt JS, Andersson M, Erjefalt I, Greiff L, Korsgren M, Linden M, Sundler F, Svensson C. Epithelium, microcirculation, and eosinophils--new aspects of the allergic airway in vivo. Allergy 1997; 52: 241-255.

31. Chen J, Zhou Y, Zhang L, Wang Y, Pepper AN, Cho SH, Kong W. Individualized Treatment of Allergic Rhinitis According to Nasal Cytology. Allergy Asthma Immunol Res 2017; 9: 403-409.

32. Kubo M. Mast cells and basophils in allergic inflammation. Curr Opin Immunol 2018; 54: 74-79.

33. Caruso M, Cibella F, Emma R, Campagna D, Tringali G, Amaradio MD, Polosa R. Basophil biomarkers as useful predictors for sublingual immunotherapy in allergic rhinitis. Int Immunopharmacol 2018; 60: 50-58. 
Siddhuraj et al. Histology-based blood leukocyte profiling in allergic rhinitis

34. Metcalfe DD, Pawankar R, Ackerman SJ, Akin C, Clayton F, Falcone FH, Gleich GJ, Irani AM, Johansson MW, Klion AD, Leiferman KM, Levi-Schaffer F, Nilsson G, Okayama Y, Prussin C, Schroeder JT, Schwartz LB, Simon HU, Walls AF, Triggiani M. Biomarkers of the involvement of mast cells, basophils and eosinophils in asthma and allergic diseases. World Allergy Organ J 2016; 9: 7.

35. Witting Christensen SK, Kortekaas Krohn I, Thuraiaiyah J, Skjold T, Schmid JM, Hoffmann HJ. Sequential allergen desensitization of basophils is non-specific and may involve p38 MAPK. Allergy 2014; 69: 1343-1349.

36. Gober LM, Eckman JA, Sterba PM, Vasagar K, Schroeder JT, Golden DB, Saini SS. Expression of activation markers on basophils in a controlled model of anaphylaxis. $J$ Allergy Clin Immunol 2007; 119: 1181-1188.

37. Salter BM, Nusca G, Tworek D, Oliveria JP, Smith SG, Watson RM, Scime T, Obminski C, Sehmi R, Gauvreau GM. Expression of activation markers in circulating basophils and the relationship to allergen-induced bronchoconstriction in subjects with mild allergic asthma. $J$ Allergy Clin Immunol 2016; 137: 936-938 e937.

38. Bergqvist A, Andersson CK, Hoffmann HJ, Mori M, Shikhagaie M, Krohn IK, Dahl R, Bjermer L, Erjefalt JS. Marked epithelial cell pathology and leukocyte paucity in persistently symptomatic severe asthma. Am J Respir Crit Care Med 2013; 188: 1475-1477. 
A

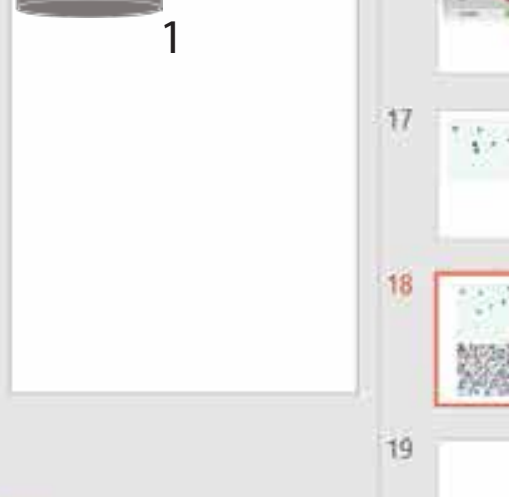

B

\begin{tabular}{|l|l|l|l|}
\hline Temperature & Time & Morphology & Antigenicity \\
\hline $70^{\circ} \mathrm{C}(\mathrm{A})$ & 10 Mins & Good & Poor \\
\hline $80^{\circ} \mathrm{C}$ (B) & 10 Mins & Good & Poor \\
\hline $97^{\circ} \mathrm{C}$ (C) & 10 Mins & Good & Poor \\
\hline $97^{\circ} \mathrm{C}$ (D) & 20 Mins & Good & Good \\
\hline
\end{tabular}

D

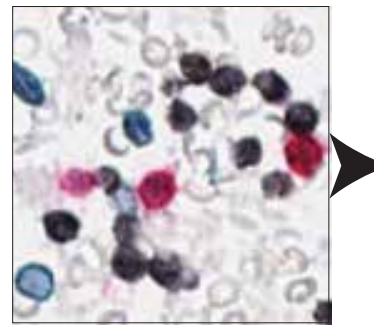

CD20 CD3 $\square$ MPO
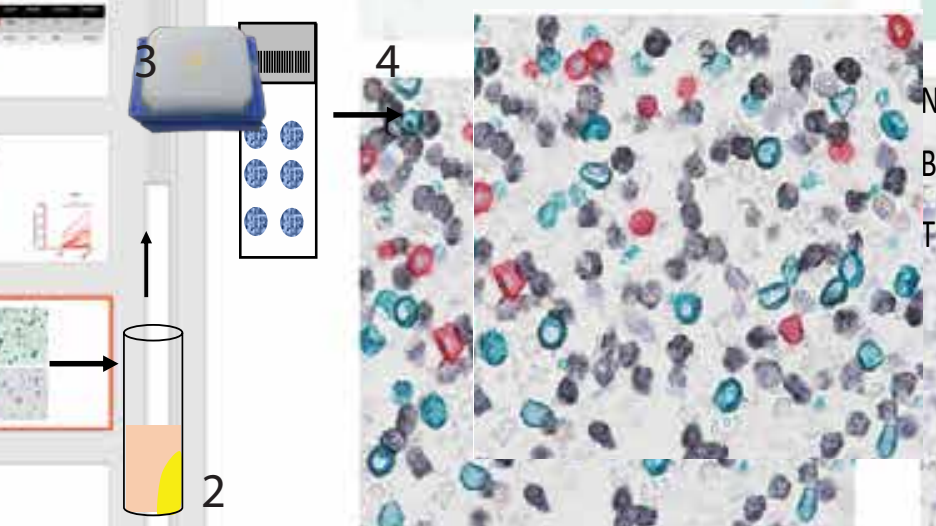

C

E

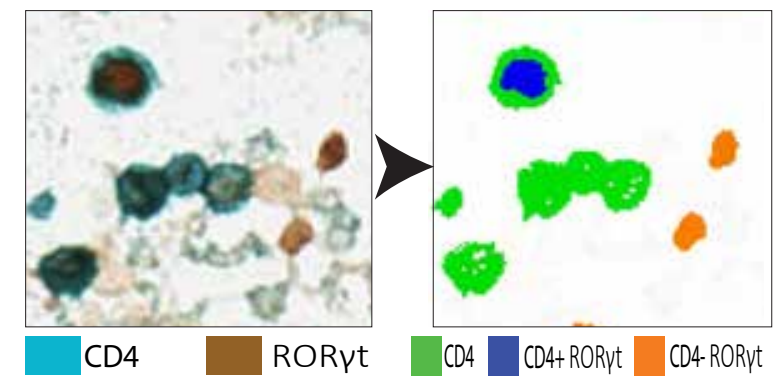


A

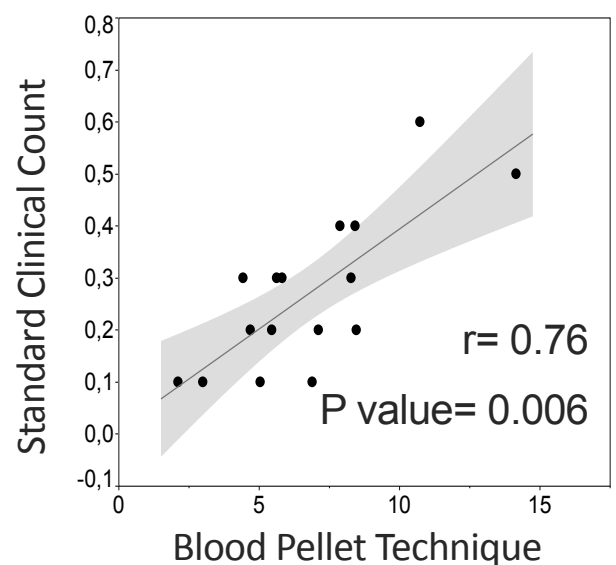

D

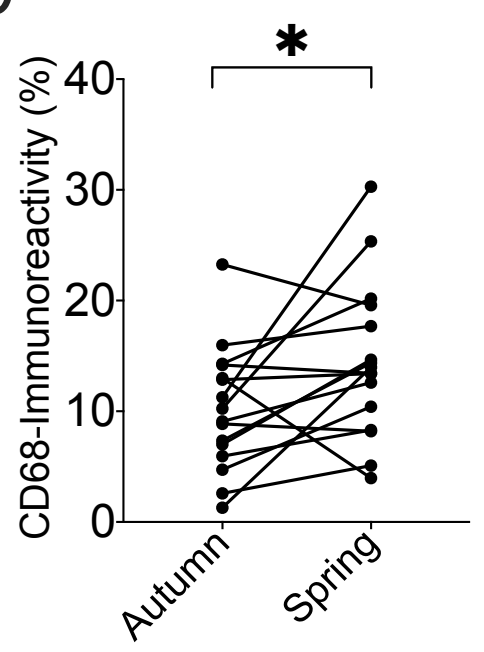

G

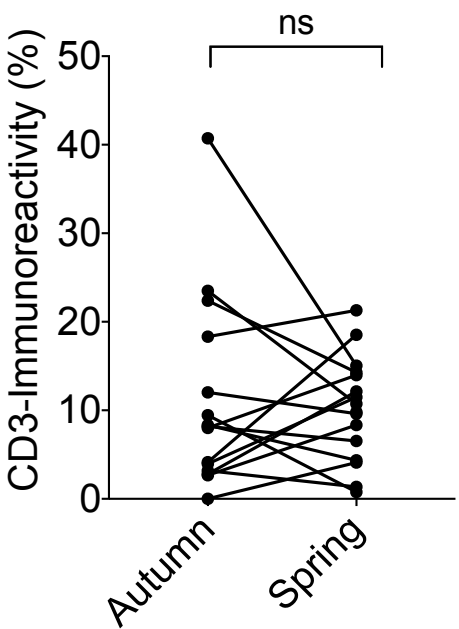

B
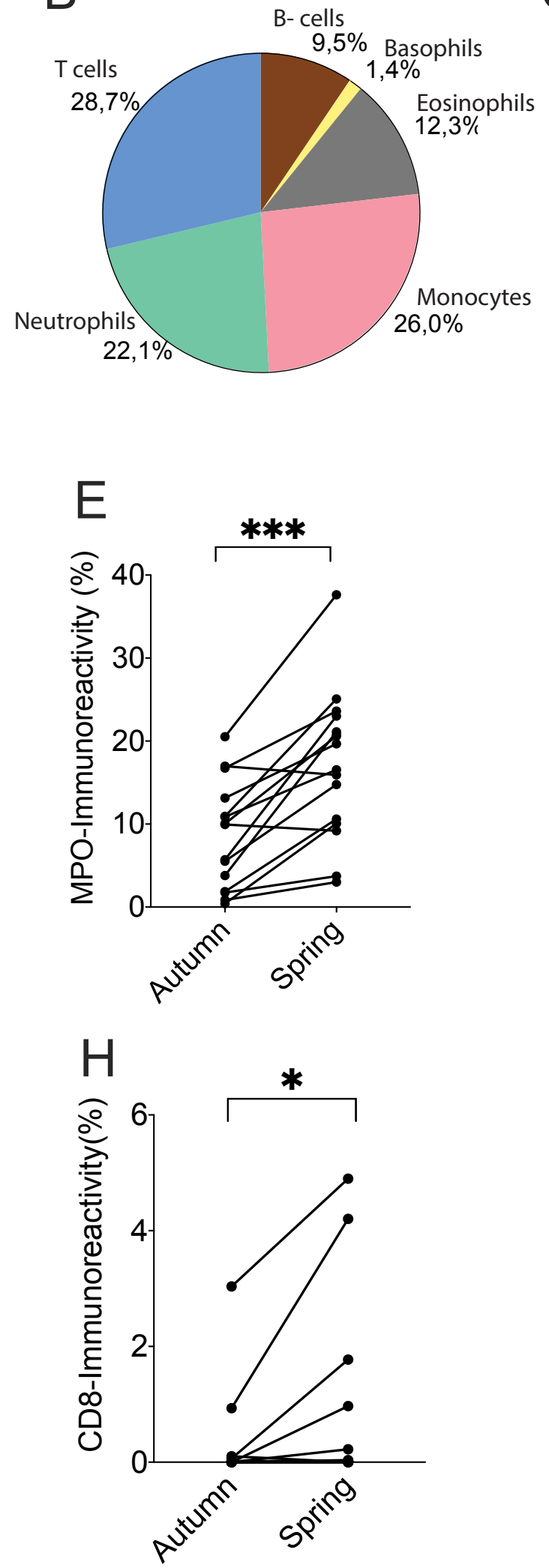

C
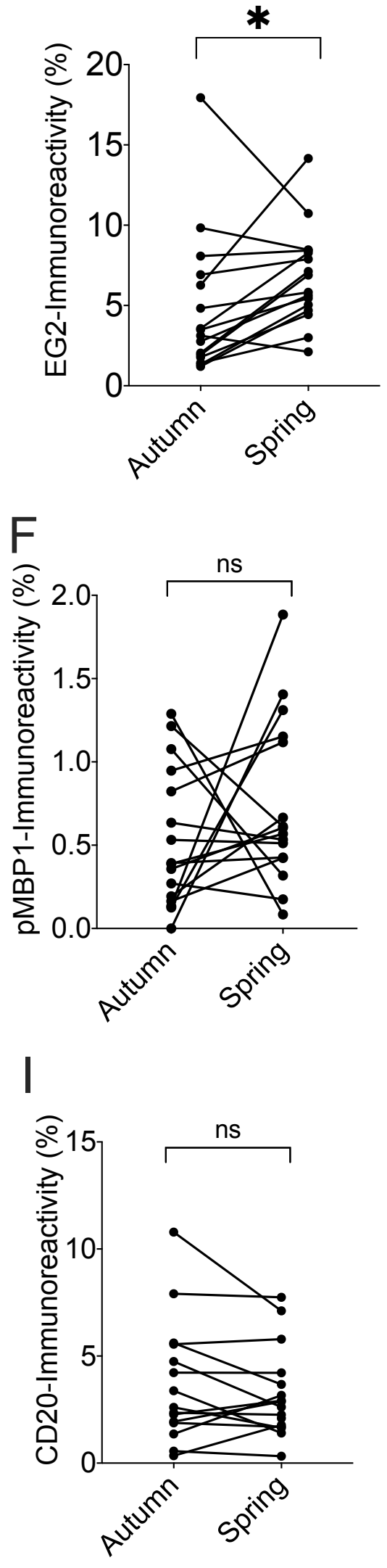


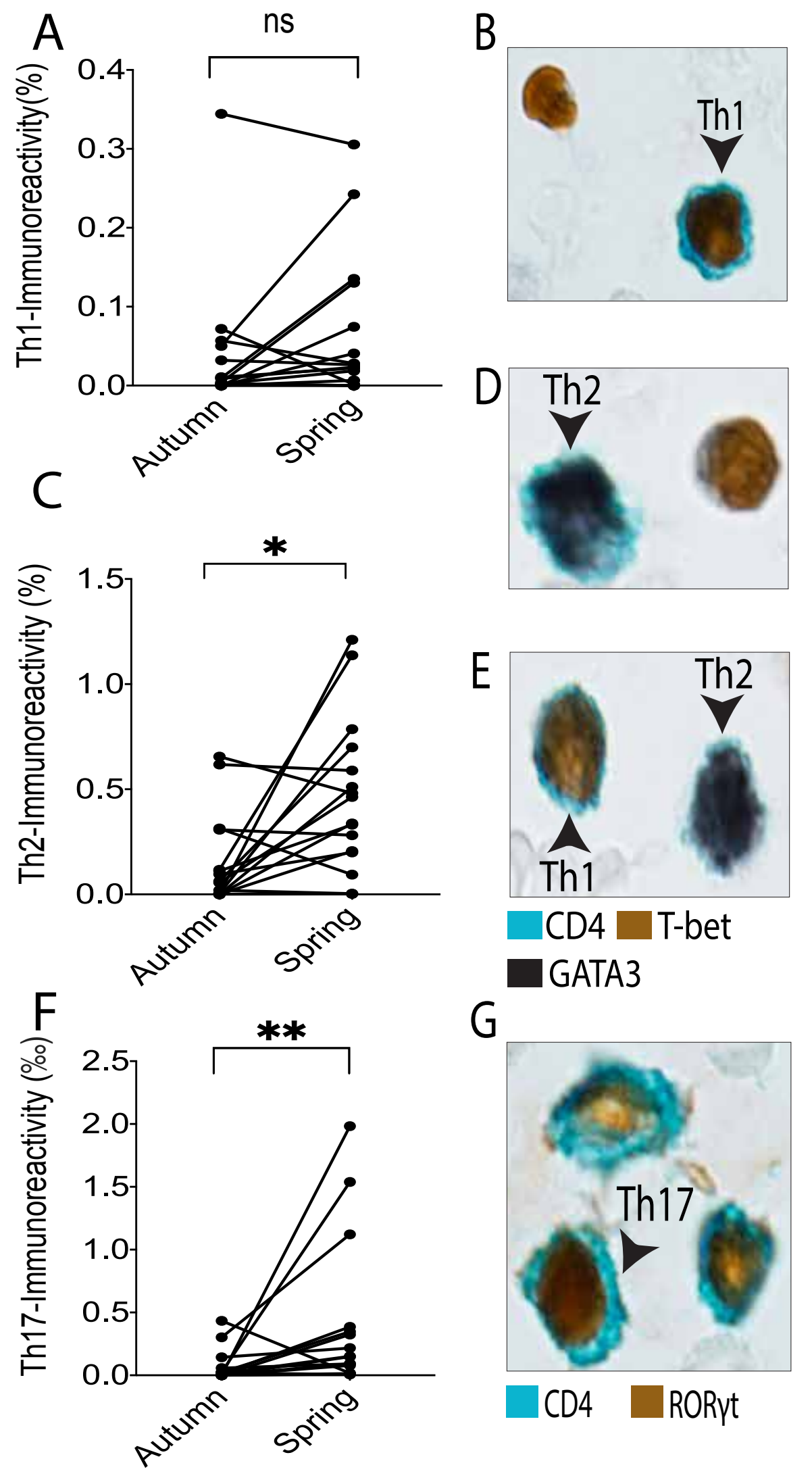


A

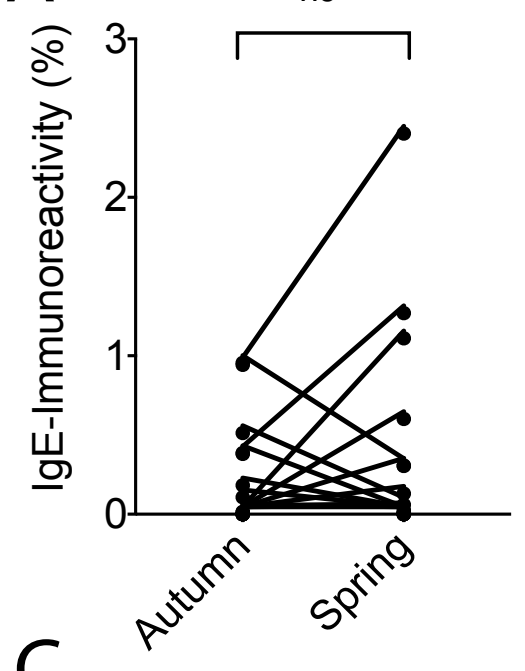

C

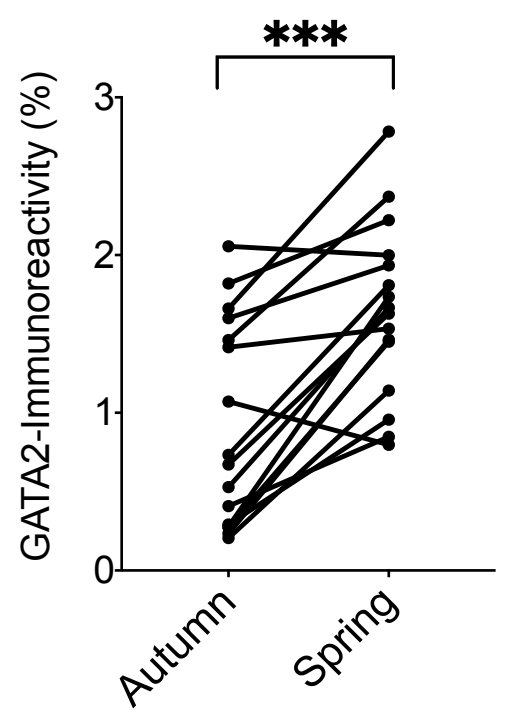

F
B

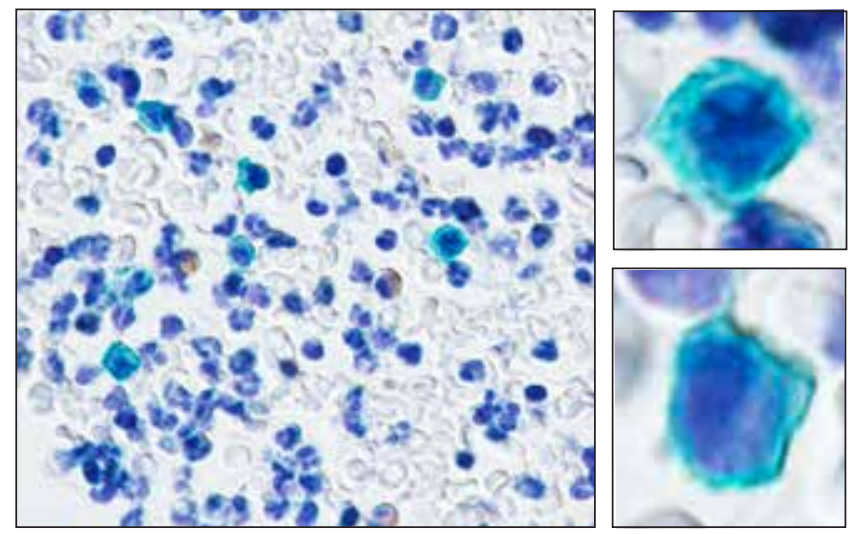

**

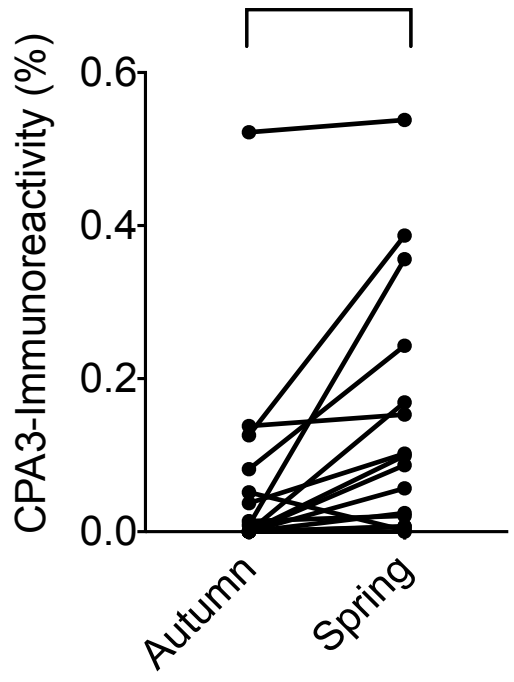

E

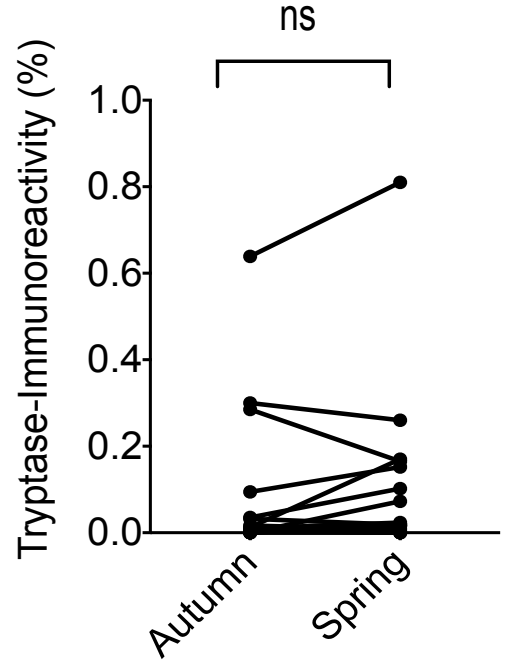

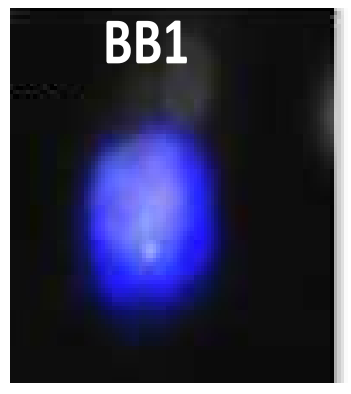
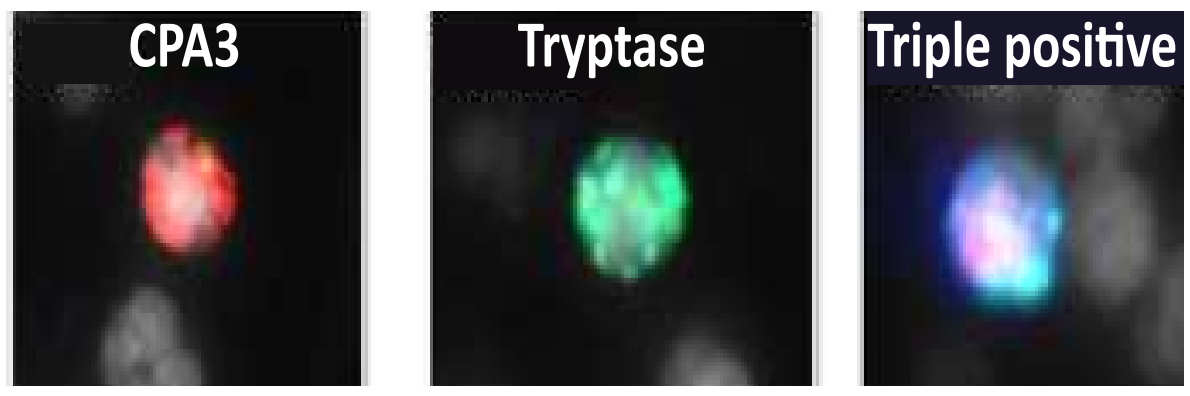

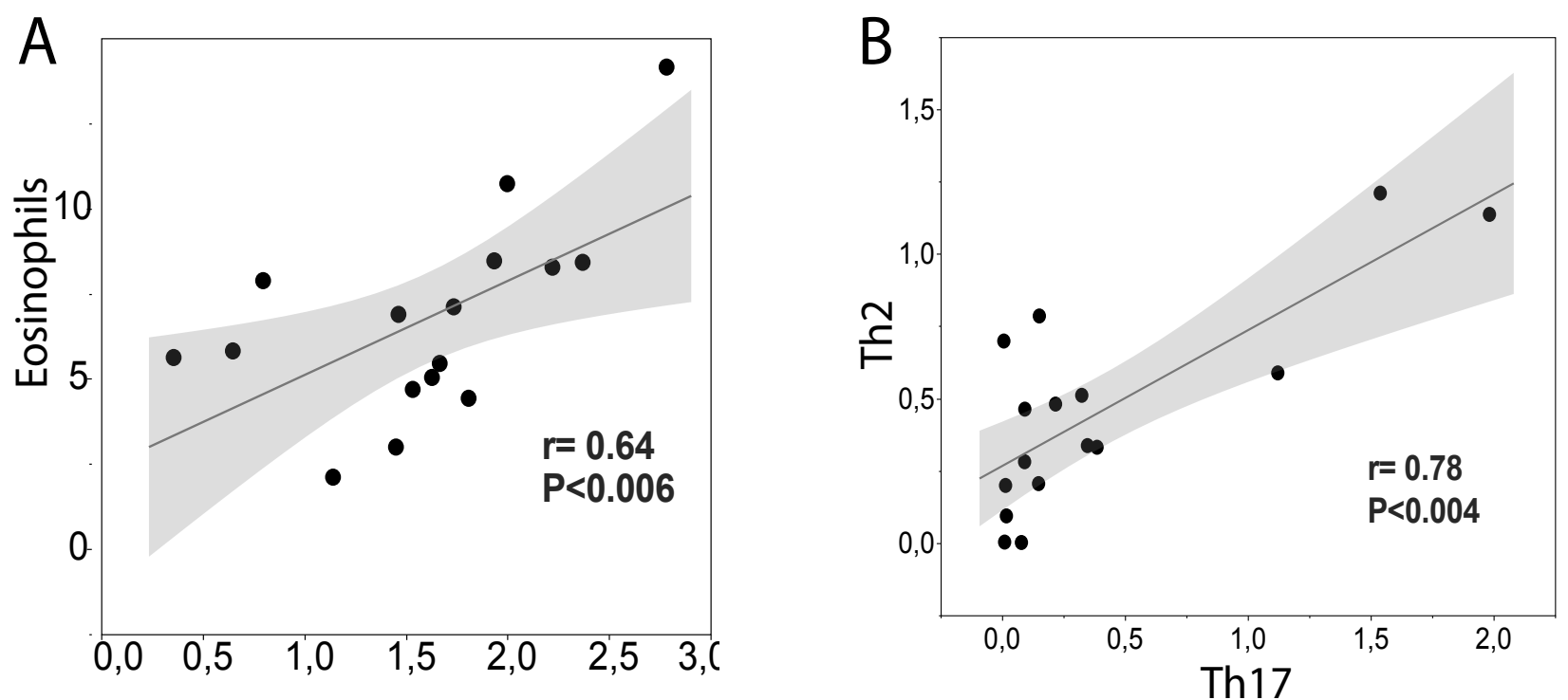


\section{Figures}

\section{Figure 1}

Schematic overview of the blood leukocyte pellet preparation (A). After blood sampling and a brief prefixation, cells are separated by dextran sedimentation (1). Layers containing leukocytes are collected and the white blood cells are spun down into pre-warmed melted agarose (2), which after being allowed to solidify and subjected to routine $4 \%$ formaldehyde fixation is embedded into paraffin (3) and cut into sections used for detection of multiple leukocyte markers by immunohistochemistry (exemplified in 4). Selection of optimal low pH heat-induced antigen retrieval conditions (B) and representative example of Htxstained leukocytes amidst the few remaining erythrocytes (C). Example of detection and segmentation strategies for computerized quantitative image analysis (D-E). In panel $E$ the brown RORyt - positive nuclei up to the left is touching CD4 immunoreactivity and is thus reclassified as a Th17 cell. 
A

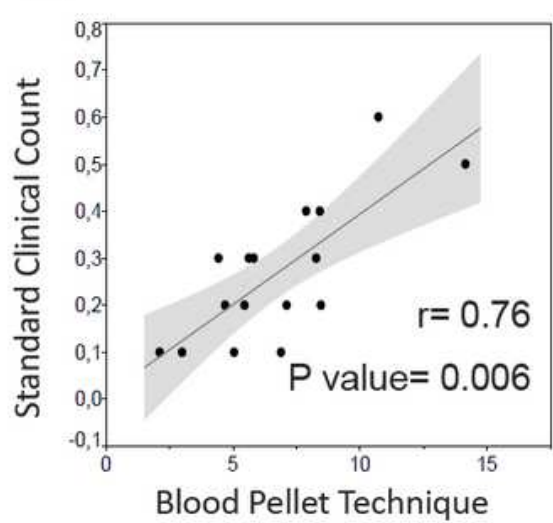

D

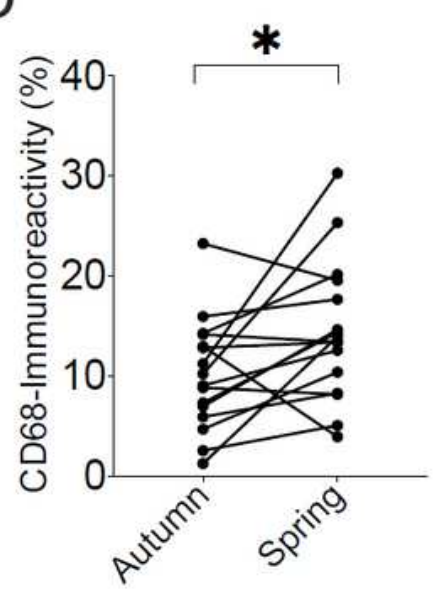

G

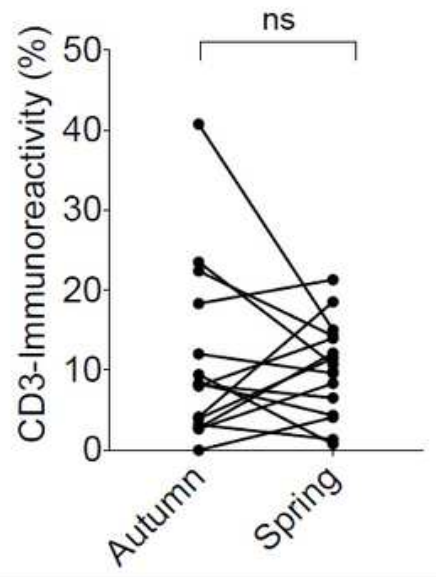

B
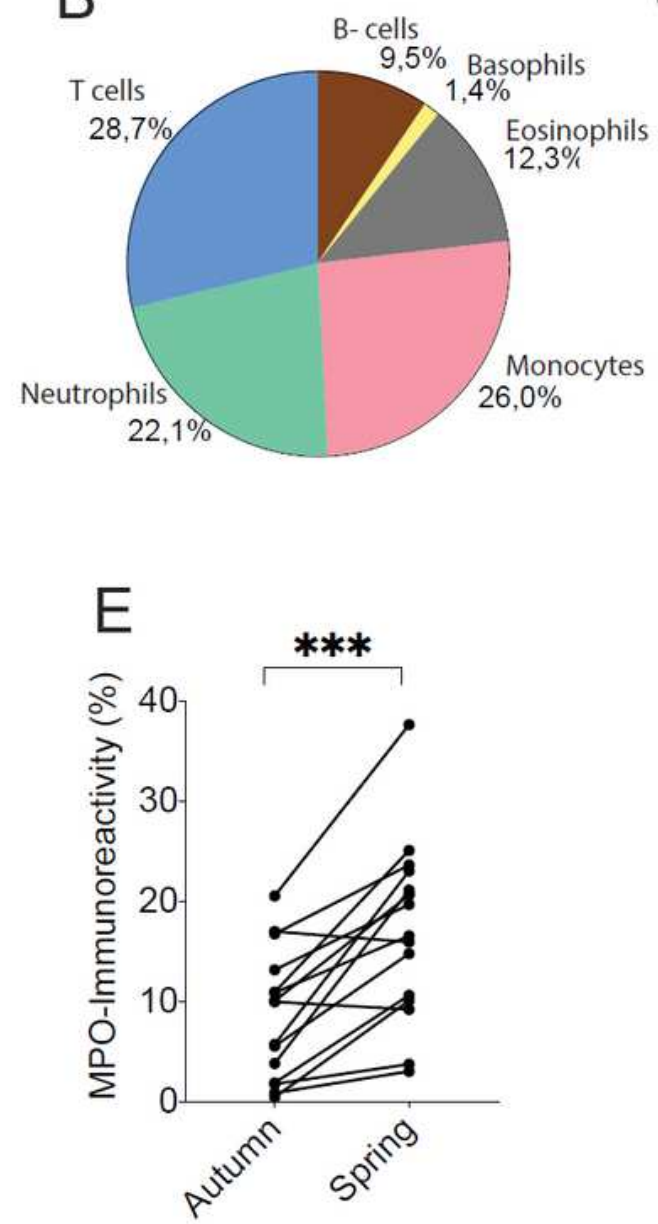

$\mathrm{H}$

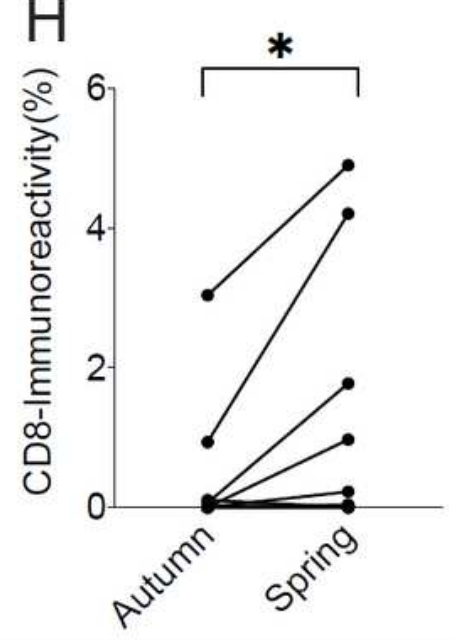

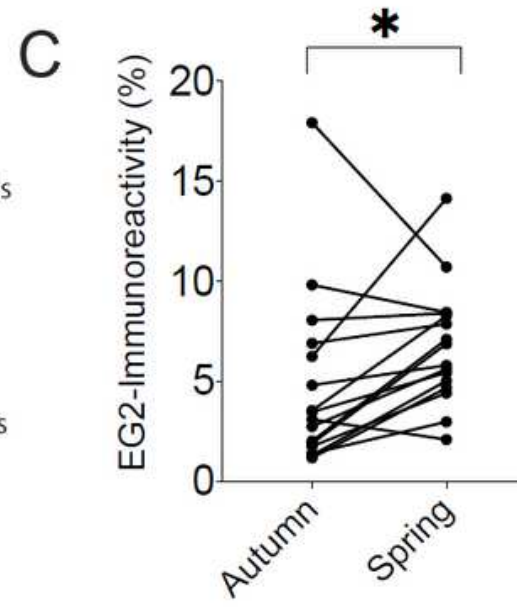

$\mathrm{F}$

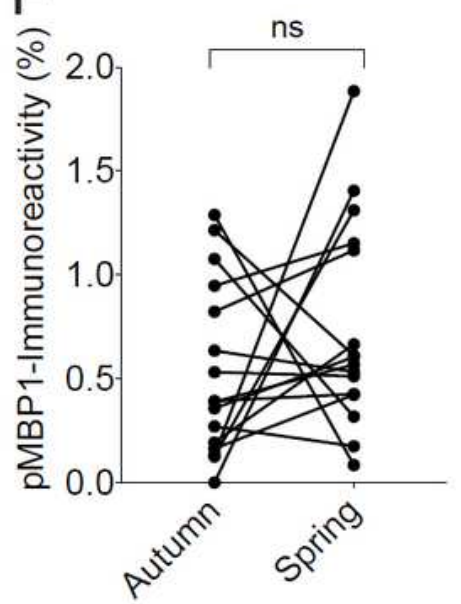

I

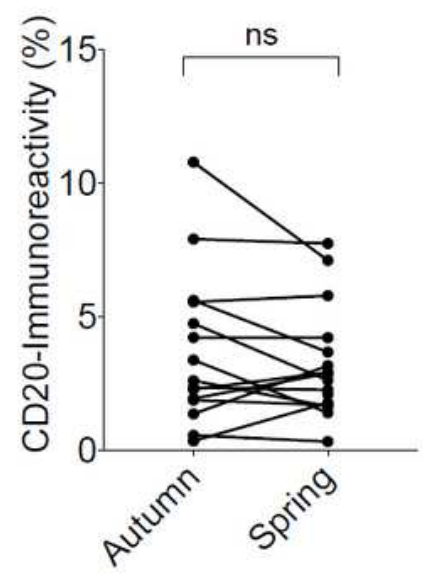

\section{Figure 2}

Correlation between clinical routine eosinophil cell count and computerized quantification of the eosinophil granule marker EG2 in blood leukocyte pellets (A). The overall composition and relative proportion of immunoreactivity for common leukocyte identity markers (B). Quantitative data of pairwise measurements of immunoreactive area in blood leukocyte pellets collected off-season/autumn and

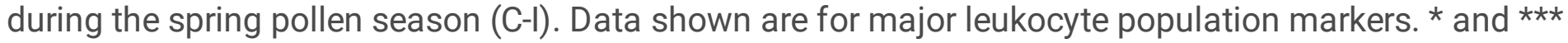


denote a significance level of $p<0.05$ and $p<0.001$, respectively. Correlation in $A$ is analyzed by Pearson correlation coefficient analysis.

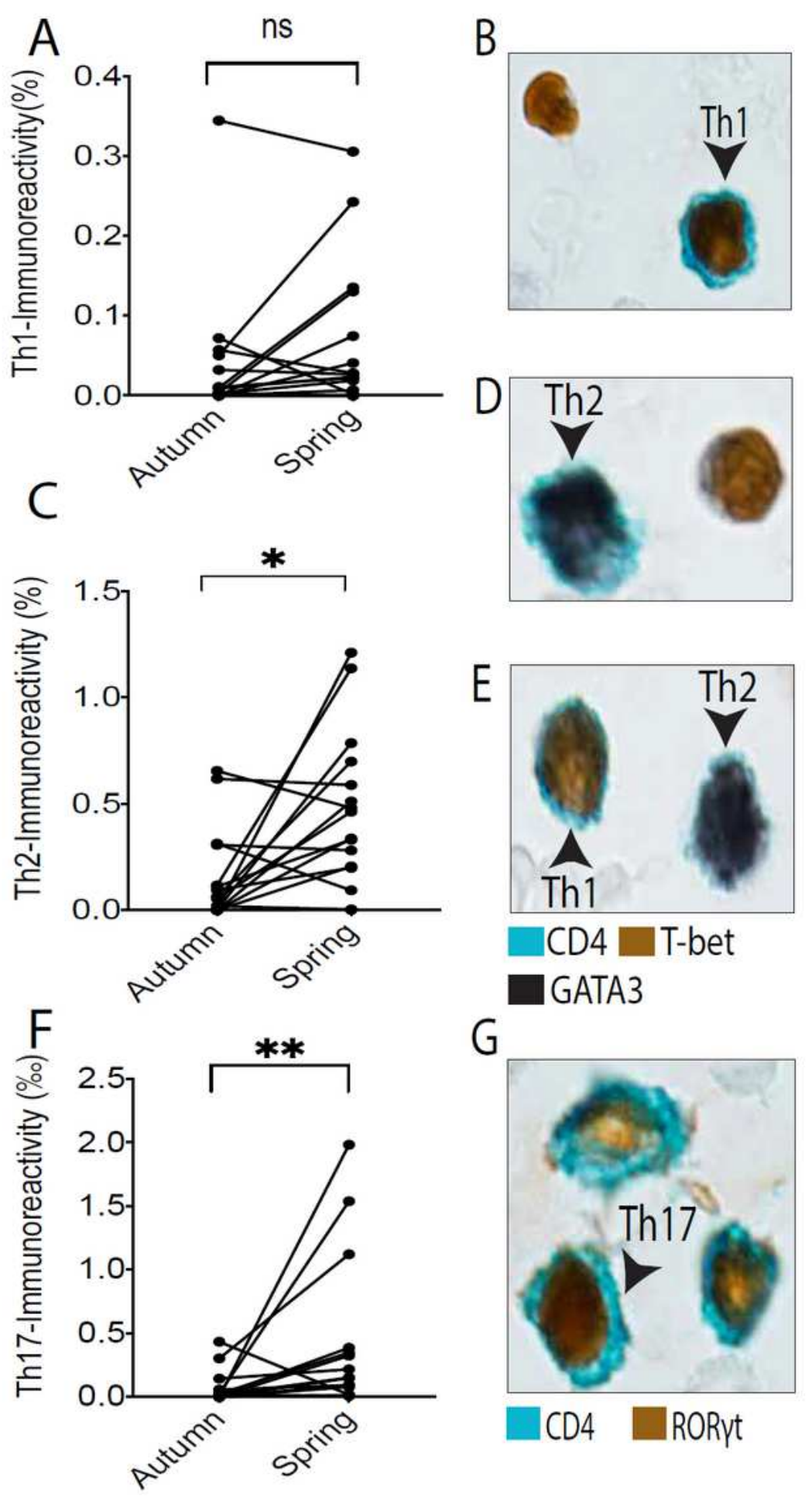

Figure 3

Quantitative T-helper subset data of pairwise autumn and spring measurements for each individual patient $(A, C, F)$. Th1, Th2, and Th17 populations were identified by double staining for CD 4 and their 
corresponding transcription factor; i.e. T-bet, GATA3 and RORYt (B, D, E,G). * and ** denote a significance level of $p<0.05$ and $p<0.01$, respectively.
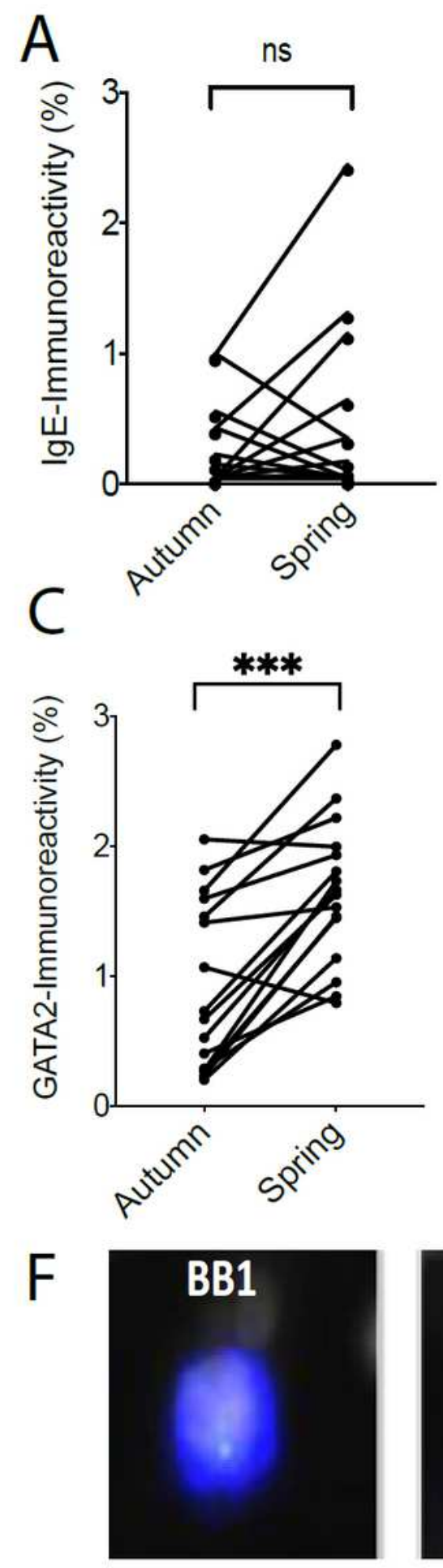

B
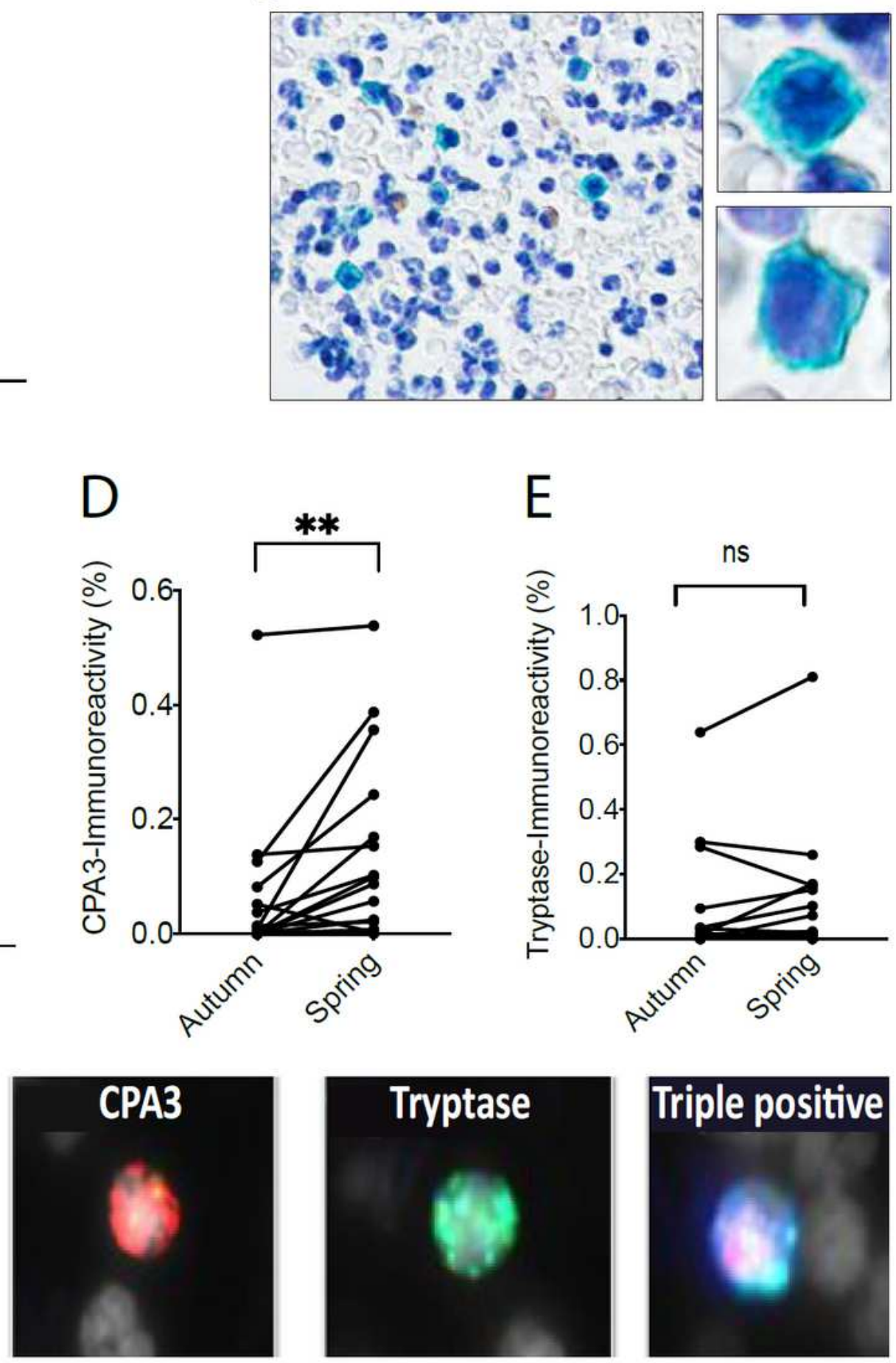
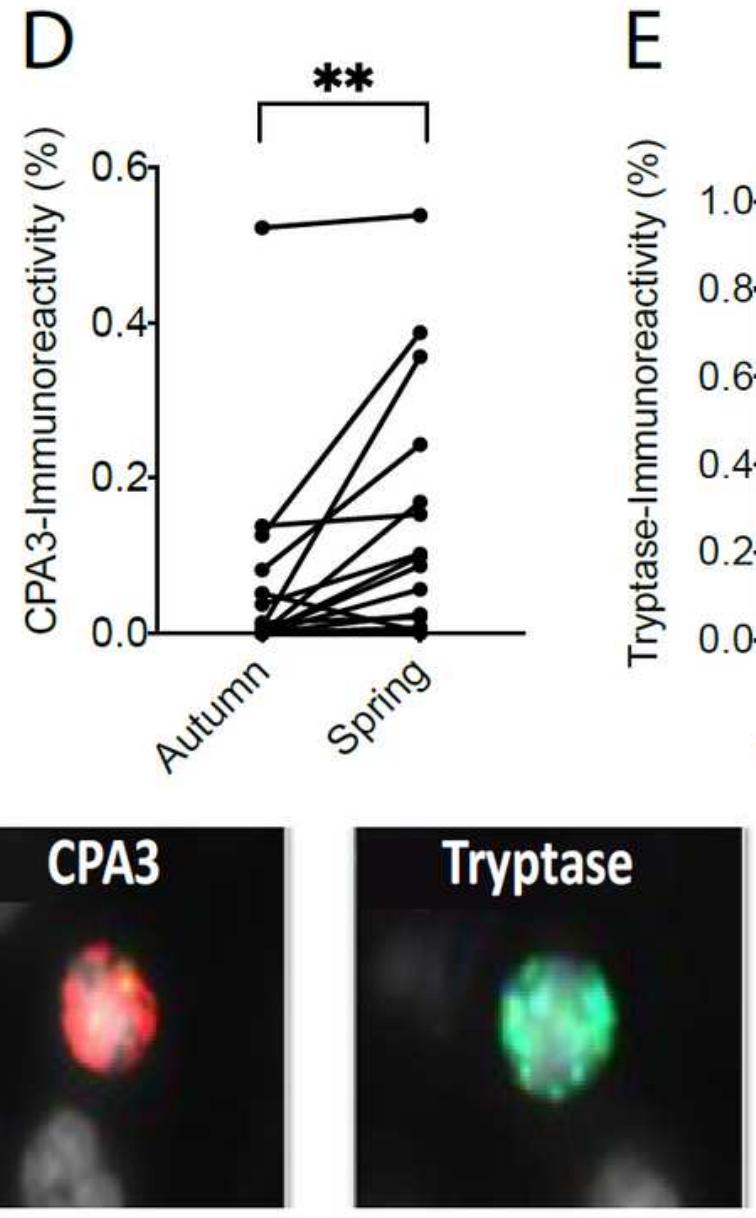

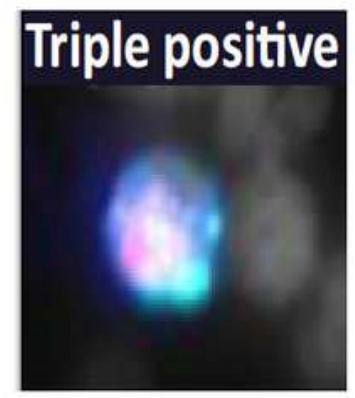

Figure 4

Pairwise leukocyte pellet data for the basophil-associated markers IgE, GATA2, CPA3 and Tryptase (A,C$E)$. ** and $* \star \star$ denote a significance level of $p<0.01$ and $p<0.001$, respectively. Representative example images of IgE-stained blood leukocytes (B). Triple immunofluorescence staining exemplifying a basophil, 
detected with the basophil marker BB1, and which is double positive for the granule proteases CPA3 and tryptase.
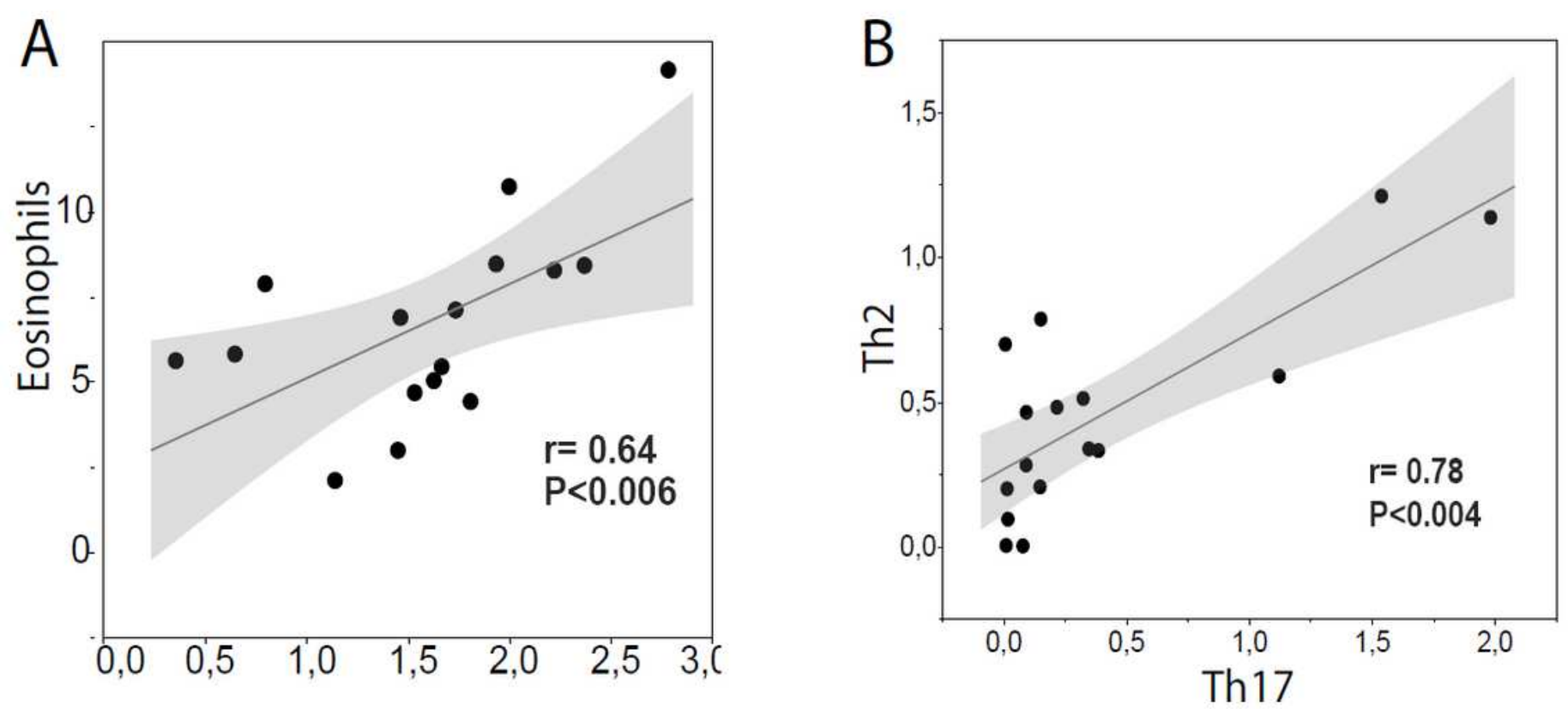

\section{Figure 5}

Scattergrams demonstrating the correlation between blood leukocyte pellet levels of eosinophils (EG2 immunoreactivity) and the basophil-associated transcription factor GATA2 ( $x$ axis in A). Correlations between blood pellet Th2 and Th17 cells during the pollen season are presented in panel $B$. The level of statistical correlation was tested with Pearson correlation coefficient analysis. 